\title{
Small World Particle Swarm Optimizer for Global Optimization Problems
}

\author{
Megha Vora and T.T. Mirnalinee \\ Department of Computer Science and Engineering \\ S.S.N College of Engineering, Anna University, Chennai, India \\ meghavora25@gmail.com, mirnalineett@ssn.edu.in
}

\begin{abstract}
Particle swarm is a stochastic optimization paradigm inspired by the concepts of social psychology and artificial intelligence. Interrelationship between individuals in a swarm is defined by the population topology, which can be depicted as a network model. Regular networks are highly clustered but the characteristic path length grows linearly with the increase in number of vertices. On the contrary, random networks are not highly clustered but they have small characteristic path length. Small world network have a distinctive combination of regular and random networks i.e., highly clustered and small characteristic path length. This paper takes forward the concept of incorporating small world theory in the Particle Swarm Optimization (PSO) framework. Efficiency of the proposed methodology is tested by applying it on twelve standard benchmark functions. Results obtained are compared with other PSO variants. Comparative study demonstrates the effectiveness of the proposed approach.
\end{abstract}

\section{Introduction}

Particle Swarm Optimization (PSO) is the global optimization technique originally proposed by Kennedy and Eberhart [1]. PSO is inspired by the paradigm of birds flocking. PSO consists of a swarm (population) of particles. Each particle flies through the multi-dimensional search space with a velocity. Velocity of a particle is updated using three terms i)previous velocity- provides necessary momentum to the particle, ii)social term- indicates how the particle is stochastically drawn towards the global best (gbest) position found so far by the swarm, and iii)cognitive term- reflects the personal thinking (pbest) of the particle. PSO has received a huge attention and popularity in the optimization community due to its algorithmic simplicity and effectiveness for solving global optimization problem. Moreover, it is computationally inexpensive in terms of both memory requirements and CPU speed. This derivative-free method is particularly suited to continuous variable problems. Even though PSO is a good and fast search algorithm, it suffers from premature convergence, especially in complex multi-peak-search problems. To overcome this problem in 2] dynamic network model is suggested; search begins with pbest and gradually incremented until it becomes gbest. In [3] Small World Particle Swarm Optimization (SWPSO) was 
proposed where gbest term in velocity updation was replaced by the small world best (SWNbest). Li et al., 4 , proposed Self Learning Particle Swarm Optimizer (SLPSO) where each particle has a set of four strategies to cope with different situations. A particle can choose an appropriate strategy at any instance according to the property of its local search space.

The population topology has a significant effect on the performance of PSO. Topology determines the way particles communicate and share information within the swarm . In [5] and 6] impact of various network topologies on particle swarm performance was studied. It was reported that path length presents a compromise between exploration and exploitation. If path length is too small, it implies that information spreads too fast, so there is a higher probability of premature convergence. On the contrary, if it is large then information takes a long time to travel through the graph. Thus, the population is more resilient and not so eager to exploit earlier on. Even a partial degree clustering (i.e., percentage of vertex's neighbours that are neighbours to one another) helps to disseminate information in the network. We therefore need to have a combination of highly clustered and small characteristic path length network. Small world network [] fits to this need of ours.

Small world concept was originally proposed by Milgram in [8] which was later modeled by Watts-Strogatz [9]. Jon Kleinberg [7] further generalized WattsStrogatz model and showed that there is a decentralized algorithm capable of finding short paths with high probability.
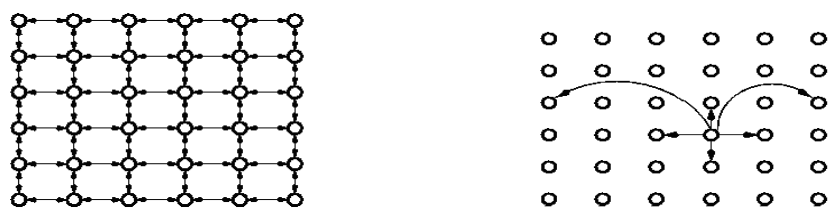

Fig. 1. Swarm of particles 6x6 Fig. 2. Small world with 2 random particles

\section{Proposed Algorithm - SWPSO-I}

In [3], small world network topology proposed by Jon Kleinberg [7] on Von Neumann network model was considered. Fig. 1 shows Von Neumann network with swarm of size $6 \times 6$ and each particle has up, down, left and right particles in its neighborhood. Now, small world network is formed by adding few random particles (in this case two) and retaining four immediate Von Neumann neighbors of a current particle. These random particles are treated as additional neighbors of current particle (see Fig. 2). The intuitive advantage of these additional random neighbors is the way small world is formed within the current swarm.

In this paper, SWPSO-I algorithm is being proposed which takes forward the above mentioned concept of SWPSO. Different from the work in [3] where inertia weight parameter was set constant, in this paper linearly decreasing inertia weight has been used. This leads to a more balanced exploration-exploitation 
trade-off [10. Moreover, in this paper particle's velocity and position is updated at individual level rather than at population level to make it adaptive. Algorithmic detail of the SWPSO-I is given below.

1 Initialize the position $\vec{X}_{k}$ and velocity $\vec{V}_{k}$ of each $k^{t h}$ particle in the swarm where $k$ varies from $(1,2, \ldots, N P) ; N P$ is the size of swarm

2 Initialize fitness counter $f e s=0$. Define maximum number of fitness evaluation $T_{-} F E s$

3 Compute the fitness $F_{k}$ for each $k^{t h}$ particle in the swarm

4 Perform steps 5 to 13 until maximum number of fitness evaluation is not exceeded

5 For each of the $k^{t h}$ particle perform steps 6 to 13

6 Compare Pbest $_{k}$ of the $k^{\text {th }}$ particle with its current fitness value $F_{k}$. If the current fitness value is better, then assign the current fitness value to pbest $_{k}$ and assign the current position to $\vec{X}_{\text {Pbest }_{k}}$

7 Compute the small world best fitness $S W N$ best $_{k}$ by selecting particle with best fitness (here minimum) in the small world neighbourhood of the $k^{t h}$ particle

8 Update the previous small world best fitness with the current value of $S W$ Nbest $_{k}$ if it is minimum. Also, update previous position of the best small world particle with the current one $\vec{X}_{S W \text { Nbest }_{k}}$

9 Update velocity of the particle using Eq. 1

$$
\vec{V}_{k}^{\prime}=\omega V_{k}+C_{1} R_{1}\left(\vec{X}_{\text {Pbest }_{k}}-\vec{X}_{k}\right)+C_{2} R_{2}\left(\vec{X}_{S W N b e s t_{k}}-\vec{X}_{k}\right)
$$

where, $\omega$ is inertia weight as defined in Eq. 2, $l b$ and $u b$ represents lower and upper bound respectively; $C_{1}$ and $C_{2}$ are acceleration constant; $R_{1}$ and $R_{2}$ are random numbers uniformly generated from the interval $[0,1]$

$$
\omega(f e s)=u b-(l b+0.1 * f e s) / T \_F E s
$$

10 If $\vec{V}_{k}^{\prime}<-V_{\max }$ then $\vec{V}_{k}^{\prime}=-V_{\max }$ else if $\vec{V}_{k}^{\prime}>V_{\max }$ then $\vec{V}_{k}^{\prime}=V_{\max }$; $V_{\max }$ is set to the half of the search range

11 Update the position of the particle using Eq. 3

$$
\vec{X}_{k}^{\prime}=\vec{X}_{k}+\vec{V}_{k}^{\prime}
$$

12 Compute the fitness of the updated particle

13 Increment the fitness counter fes ++

\section{$3 \quad$ Experimental Results}

\subsection{Benchmark Functions Used}

For evaluating the performance of SWPSO-I a test set of twelve functions is used. Details of the functions are given in Table 1. The test function set includes the traditional functions and traditional functions with noise, shift and rotation as described in [11. Detail description of the Shifting, rotation and noise addition method for the test functions $f_{8}-f_{12}$ can be found in [11. 
Table 1. Test functions, where $f_{\min }$ is the minimum value of a function and $\mathrm{S} \epsilon R_{n}$

\begin{tabular}{|l|l|c|c|}
\hline Name & Test Function & Search Range $S$ & $f_{\min }$ \\
\hline Sphere & $f_{1}(x)=\sum_{i=1}^{n} x_{i}^{2}$ & {$[-100,100]$} & 0 \\
\hline Rastrigin & $f_{2}(x)=\sum_{i=1}^{n}\left(x_{i}^{2}-10 \cos \left(2 \Pi x_{i}\right)+10\right)$ & {$[-5.12,5.12]$} & 0 \\
\hline Griewank & $f_{3}(x)=\frac{1}{4000} \sum_{i=1}^{n}\left(x_{i}-100\right)^{2}-\prod_{i=1}^{n} \cos \frac{x_{i}-100}{\sqrt{i}+1}$ & {$[-600,600]$} & 0 \\
\hline Ackley & $f_{4}(x)=-20 \exp \left(-0.2 \sqrt{\left.\frac{1}{n} \sum_{i=1}^{n} x_{i}^{2}\right)-}\right.$ & & \\
& $\exp \left(\frac{1}{n} \sum_{i=1}^{n} \cos \left(2 \pi x_{i}\right)\right)+20+e$ & {$[-3232]$} & 0 \\
\hline Rosenbrock & $f_{5}(x)=\sum_{i=1}^{n} 100\left(x_{i+1}^{2}-x_{i}\right)^{2}+\left(x_{i}-1\right)^{2}$ & {$[-2.048,2.048]$} & 0 \\
\hline Schwefel-1-2 & $f_{6}(x)=\sum_{i=1}^{n}\left(\sum_{j=1}^{i} x_{j}^{2}\right)$ & {$[-100,100]$} & 0 \\
\hline Schwefel-2-22 & $f_{7}(x)=\sum_{i=1}^{n}\left|x_{i}\right|+\prod_{i=1}^{n}\left|x_{i}\right|$ & {$[-10,10]$} & 0 \\
\hline S-Sphere & $f_{8}(x)=\operatorname{CEC} 05\left(F_{1}\right)$ & {$[-100,100]$} & -450 \\
\hline S-Rosenbrock & $f_{9}(x)=\operatorname{CEC} 05\left(F_{6}\right)$ & {$[-100,100]$} & 390 \\
\hline S-schwefel-1-2 & $f_{10}(x)=\operatorname{CEC} 05\left(F_{2}\right)$ & {$[-100,100]$} & -450 \\
\hline S-schwefel-1-2-Noisy & $f_{11}(x)=\mathrm{CEC} 05\left(F_{4}\right)$ & {$[-100,100]$} & -450 \\
\hline RS-Ackely & $f_{12}(x)=\mathrm{CEC} 05\left(F_{8}\right)$ & {$[-32,32]$} & -140 \\
\hline
\end{tabular}

\subsection{Simulation Strategy}

To fairly compare the results obtained by SWPSO-I with SWPSO [3] and already published results of SLPSO 4], simulation was done considering a swarm of size of $20(4 \times 5)$. Swarm was initialized in the search space using symmetric initialization strategy (i.e., uniformly distributed over the search space S). Functions were tested in 30-dimension (30D) with 30 independent runs. Each run of the algorithm is terminated when the number of function evaluations (T_FEs) exceed $1 \mathrm{e}+05$. Acceleration coefficient parameter $C_{1}$ and $C_{2}$ is set to 1.494 . These values were chosen to ensure good convergence [12. Inertia weight parameter $\omega$ is varied linearly from 0.9 to 0.4 according to the Eq. 2 for both SWPSO-I and SLPSO, while it is set to 0.72 for SWPSO. The larger step size at the beginning offers a better exploration and the smaller step size towards the end offers better exploitation, thus decreasing chances of missing a global optima. For SWPSO and SWPSO-I two small world randomized particles were chosen. Experiments with different number (1,2 and 3) of randomized particles showed that using two randomized particles convergence to optimal solution was faster.

\subsection{Results on Benchmark Functions}

Table 2 shows the mean and standard deviation (within parentheses) for 30 runs for three algorithms. Results in bold face indicate the best ones. Table 3 shows the result of two tailed unpaired t-tests [13] with 58 degree of freedom between the best algorithm and the second best in each case.

It can be observed from Table 2 that SWPSO-I outperforms SWPSO in terms of mean and standard deviation. Only in one case $\left(f_{7}\right)$ SWPSO-I mean value is larger, but Table 3 shows that this difference is not statistically significant. For the function $f_{12}$ both SWPSO-I and SWPSO have same mean value but low standard deviation value of SWPSO-I shows that SWPSO-I is more stable than SWPSO. Thus in terms of stability and accuracy SWPSO-I outperforms 
Table 2. Comparison results of mean and standard deviation (within parentheses) in 30 Dimensions over 30 runs

\begin{tabular}{|c|c|c|c|c|}
\hline$f$ & $f_{1}$ & $f_{2}$ & $f_{3}$ & $f_{4}$ \\
\hline SWPSO-I & $9.6 \mathrm{e}-71(5.2 \mathrm{e}-70)$ & $70.64(15.13)$ & $6.56 e-3(8.91 e-3)$ & $5.35 \mathrm{e}-1(7.63 \mathrm{e}-1)$ \\
\hline SWPSO & $2.94 \mathrm{e}-61(1.04 \mathrm{e}-60)$ & $69.52(20.59)$ & $9.3 \mathrm{e}-3(1.08 \mathrm{e}-2)$ & $1.452(1.086)$ \\
\hline SLPSO & $2.78 \mathrm{e}-50(8.09 \mathrm{e}-49)$ & $\mathbf{0}(0)$ & $0.227(0.144)$ & $3.47 \mathrm{e}-14(4.45 \mathrm{e}-14)$ \\
\hline$f$ & $f_{5}$ & $f_{6}$ & $f_{7}$ & $f_{8}$ \\
\hline SWPSO-I & $6.2(\mathbf{5 . 3})$ & $5.11 \mathrm{e}-4(1.01 \mathrm{e}-3)$ & $1.75 \mathrm{e}-27(9.48 \mathrm{e}-27)$ & $3.67 \mathrm{e}-13(7.09 \mathrm{e}-13)$ \\
\hline SWPSO & $10.68(12.75)$ & $1.66 \mathrm{e}+2(9.12 \mathrm{e}+2)$ & $1.51 \mathrm{e}-29(8.29 \mathrm{e}-29)$ & $1.79 \mathrm{e}+2(9.85 \mathrm{e}+2)$ \\
\hline SLPSO & $2.06(12.3)$ & $7.93 \mathrm{e}-3(0.233)$ & $1.35 \mathrm{e}-26(3.88 \mathrm{e}-25)$ & $1.00 \mathrm{e}-13(1.74 \mathrm{e}-13)$ \\
\hline$f$ & $f_{9}$ & $f_{10}$ & $f_{11}$ & $f_{12}$ \\
\hline SWPSO-I & $23.6(\mathbf{4 7 . 0 5})$ & $2.06 \mathrm{e}-3(7.69 \mathrm{e}-3)$ & $1.46 e+3(9.73 e+2)$ & $20.9(6.82 e-2)$ \\
\hline SWPSO & $1.15 \mathrm{e}+10(1.59 \mathrm{e}+8)$ & $8.40(3.24 \mathrm{e}+1)$ & $4.32 \mathrm{e}+3(4.95 \mathrm{e}+3)$ & $20.9(0.0860)$ \\
\hline SLPSO & $\mathbf{1 0 . 7}(140)$ & $1.4 \mathrm{e}-6(1.57 \mathrm{e}-6)$ & $1.16 \mathrm{e}+4(2.99 \mathrm{e}+4)$ & $\mathbf{2 0 . 4}(0.846)$ \\
\hline
\end{tabular}

SWPSO. Table 2 shows that for seven cases SWPSO-I gives higher mean (i,e, worse) than the mean of SLPSO algorithm; Table 3 shows that this difference is statistically not significant for five cases. Moreover for $f_{12}$ function difference in mean values of SWPSO-I and SLPSO is not significant but from standard deviation point of view SWPSO-I is giving more stable result. Similar is the case for function $f_{9}$ and $f_{5}$. Thus SWPSO-I is more stable compared to SLPSO.

Table 3. Results of unpaired t-tests on the data of Table 2

\begin{tabular}{|c|c|c|c|l|}
\hline Function & Std. Error & $\mathrm{t}$ & Two tailed $\mathrm{p}$ & Significance \\
\hline $\mathrm{f} 1$ & 0 & 1.548 & 0.1270 & Not significant \\
\hline $\mathrm{f} 2$ & 3.759 & 18.493 & $<0.0001$ & Extremely significant \\
\hline $\mathrm{f} 3$ & 0.003 & 1.071 & 0.2882 & Not significant \\
\hline f4 & 0.026 & 8.368 & $<0.0001$ & Extremely significant \\
\hline f5 & 2.445 & 1.693 & 0.0958 & Not quite significant \\
\hline f6 & 0.043 & 0.174 & 0.8622 & Not significant \\
\hline f7 & 0.000 & 1.002 & 0.3203 & Not significant \\
\hline f8 & 0.0000 & 2.003 & 0.0498 & Not significant \\
\hline f9 & 26.965 & 0.478 & 0.6342 & Not significant \\
\hline f10 & 0.001 & 1.466 & 0.1480 & Not significant \\
\hline f11 & 922.537 & 3.097 & 0.0030 & Very significant \\
\hline f12 & 0.155 & 3.226 & 0.0021 & Very significant \\
\hline
\end{tabular}

Table 4. Summarized t-test results

\begin{tabular}{|l|c|c|c|}
\hline $\begin{array}{l}\text { SWPSO-I } \\
\text { Vs. }\end{array}$ & $\begin{array}{c}\text { Significantly } \\
\text { better }\end{array}$ & $\begin{array}{c}\text { Significantly } \\
\text { worse }\end{array}$ & $\begin{array}{c}\text { Statistically } \\
\text { equivalent }\end{array}$ \\
\hline SWPSO & 3 & 0 & 9 \\
\hline SLPSO & 2 & 2 & 8 \\
\hline
\end{tabular}


Table 4 summarizes t-test results. It is apparent from the table that performance of SWPSO-I is better than SWPSO and comparable with that of SLPSO.

\section{Conclusions}

In this paper, we have proposed SWPSO-I algorithm which takes forward the concept of SWPSO. To evaluate the performance of the proposed algorithm we have tested it on 12 benchmark functions. Mean, standard deviation and t-test are the performance metrics used for evaluation. Comparative study of the proposed algorithm with SWPSO and SLPSO justify the efficiency of the proposed SWPSO-I. Applying SWPSO-I for real world optimization problems and molding it for data clustering and image segmentation will be the future scope of study.

\section{References}

1. Kennedy, J., Eberhart, R.C.: Particle swarm optimization. In: IEEE International Conference on Neural Networks, vol. 4, pp. 1942-1948 (1995)

2. Suganthan, P.N.: Particle swarm optimizer with neighborhood operator. In: Congress on Evolutionary Computation, pp. 1958-1962 (1999)

3. Saxena, A.K., Vora, M.: Novel approach for the use of small world theory in particle swarm optimization. In: 16th International Conference on Advanced Computing and Communications, pp. 363-366. IEEE (2008)

4. Li, C., Yang, S., Nguyen, T.: A self-learning particle swarm optimizer for global optimization problems. IEEE Transactions on Systems, Man, and Cybernetics, Part B 42(3), 627-646 (2012)

5. Mendes, R.: Population Topologies and Their Influence in Particle Swarm Performance. PhD thesis, University of Minho (2004)

6. Kennedy, J.: Small worlds and mega-minds: Effects of neighborhood topology on particle swarm performance. In: IEEE CEC, pp. 1931-1938 (1999)

7. Kleinberg, J.: The small-world phenomenon: An algorithmic perspective. Technical report, Cornell University Ithaca, NY, USA (1999)

8. Milgram, S.: The small world problem. Psychology Today 2, 60-67 (1967)

9. Watts, D., Strogatz, S.: Collective dynamics of small-world networks. Nature 393, 440-442 (1998)

10. Eberhart, R.C., Shi, Y., Kennedy, J.: Swarm Intelligence. Morgan Kaufmann (2001)

11. Suganthan, P.N., Hansen, N., Liang, J.J., Deb, K., Chen, Y.P., Auger, A., Tiwari, S.: Problem definitions and evaluation criteria for the cec 2005 special session on real-parameter optimization. Technical report (2005)

12. van den Bergh, F.: An Analysis of Particle Swarm Optimizer. PhD thesis, Department of Computer Science, University of Petoria, South Africa (2002)

13. Seltman, H.J.: Experimental Design and analysis. Carnegie Mellon University (2012) 